\title{
Retrospective Cohort Study Evaluating Clinical Outcomes in Lower Extremity Ulcers Treated with a Bi-layered Bioengineered Skin Substitute (BBSS) as Compared to Standard Therapy
}

\author{
Min Yao, Latricia Allen, Kirsti Diehl, Michael French, Nanjin Park and Vickie R Driver* \\ PVAMC Department of Surgery, VA New England Health Care Division, BLDG 32 Research Center,830 Chalkstone Avenue, Providence, RI 02908, USA \\ *Corresponding author: Vickie R Driver, VA New England Health Care Division, PVAMC Department of Surgery, BLDG 32 Research Center,830 Chalkstone Avenue, \\ Providence, RI 02908, USA, Tel: +1 401-273-7100 ; Fax: +1 401-273-7100; E-mail: drvdriver@aol.com
}

Rec date: November 21, 2016; Acc date: December 21, 2016; Pub date: December 28, 2016

Copyright: ( 2016 Yao M et al. This is an open-access article distributed under the terms of the Creative Commons Attribution License, which permits unrestricted use, distribution, and reproduction in any medium, provided the original author and source are credited.

\begin{abstract}
Purpose/Background: The purpose of this retrospective cohort study was to evaluate the efficacy of a bi-layered bioengineered skin substitute (BBSS) on wound healing in patients with chronic lower extremity ulcers (LEUs) and multiple co-morbidities such as diabetes with neuropathy, hypertension, cardiovascular, chronic kidney disease, peripheral vascular disease, critical limb ischemia and venous disease.
\end{abstract}

Methods: A retrospective cohort study was conducted using the Boston University Medical Center electronic medical record and clinical warehouse data. Co-morbidities such as hypertension, coronary heart disease, cerebrovascular disease, peripheral arterial disease, chronic renal disease, congestive heart failure, and history of minor and major amputation were examined to assess any correlation with wound healing.

Results: 158 BBSS and 126 control patients with LEUs were included in this cohort study with a follow-up period of 180 days. More ulcers healed in the BBSS group than in the control group. The rate for complete wound closure was $69.84 \%$ in BBSS and $41.98 \%$ in the control $(P<0.05)$. Average time to achieve wound closure was 70.1 days in the BBSS group and 118.03 days in the control group $(P<0.05)$. The BBSS group was 1.69 times $(95 \% \mathrm{Cl}=1.14-2.73)$ more likely to have their wound healed as compared to the control patients.

Conclusion: BBSS may be useful in accelerating wound healing in patients with severe LEUs.

Keywords: Bioengineered skin substitute; Wound healing

\section{Introduction}

Ulcers or open wounds of the lower extremity are a major health problem that can significantly decrease patient quality of life, often leading to prolonged hospitalization and amputation [1]. BBSS have been used in recent years to treat complicated non-healing diabetic and venous stasis ulcers. The BBSS used in this study is created by culturing human foreskin derived neonatal fibroblasts in a bovine type I collagen matrix which epidermal keratinocytes are cultured and allowed to stratify allowing both cells and matrix to the non-healing wound [2]. BBSS has been reported as being able to promoting wound healing and prevent complications in LEUs via randomized clinical trials (RCTs) [3-5]. RCTs are considered to be the gold standard in generating major evidence for a clinical intervention [6]. However, patients with chronic wounds can be clinically complex with compliance challenges and often present with multiple co-morbidities, as well as varying underlying etiologies. RCT proven therapies may fail to be effective in clinically complex patients. This might be attributed to RCT designs that do not reflect the complex issues faced in clinical practice and are limited by the challenges of designing such studies given wound heterogeneity. Use of practice based evidence (PBE) and observational study data can provide important clinical insights that are generally missing from RCTs [7-10].
Therefore, the purpose of this study is to evaluate the efficacy of BBSS use in the treatment of complex wounds as compared to patients with wounds treated without BBSS.

\section{Materials and Methods}

This was an Institutional Review Board (IRB) approved retrospective cohort study, which examined administrative electronic medical record (EMR) data at the Boston Medical Center. The study cohort included patients with LEUs, who were treated with or without BBSS. Patients with LEUs were identified via longitudinal medical records from the Boston University Medical Center (BUMC) data warehouse.

Data retrieved from the BUMC data warehouse consisted of: demographics, International Classification of Diseases Codes (ICD-9), and Current Procedural Terminology Codes (CPT), and additional information (i.e., ulcer history, ulcer start date, ulcer end date, ulcers size, ulcer grade, history of LEUs, amputation, and BBSS application date) were captured through chart review of BUMC electronic medical records by trained clinical medical professionals.

Inclusion criteria for this study consisted of patients who had: received clinic treatment by either a podiatric or vascular surgeon, had a diagnostic code in their medical record for diabetes or peripheral arterial disease, and at least one diagnosis code for ulcer in their medical record, or the word "ulcer" or "amputation" was documented 
Citation: Yao M, Allen L, Diehl K, French M, Park N, Driver VR (2016) Retrospective Cohort Study Evaluating Clinical Outcomes in Lower Extremity Ulcers Treated with a Bi-layered Bioengineered Skin Substitute (BBSS) as Compared to Standard Therapy. Gen Med (Los Angeles) 4: 282. doi:10.4172/2327-5146.1000282

Page 2 of 6

in an outpatient medical record note, discharge summary, operations report, or visiting nurse report.

Subjects were excluded based on CPT and ICD-9 codes for the following conditions: HIV positive status, traumatic injury, burn injury, critical limb ischemia, sickle cell disease, cancer history, liver failure or age less than 18 years. Additionally, subjects with any of these noted conditions found during chart review were also excluded. No exclusion was made based on Wagner wound grade or severity.

Paper records of BBSS orders were utilized to obtain a list of patient cases going back to 2006. Chart review was performed to confirm when a patient received an application of BBSS for wound treatment. Additional BBSS patient cases were selected based on a finding of at least one of the following words, "Apligraf, Apligraph, Bilayered skin substitute, BBSS" in the outpatient notes, discharge summaries, operations reports or visiting nurse reports. Controls were matched to case subjects based on age (within 5 years) and gender. The controls were noted by the lack of use of BBSS, which was confirmed via chart review. Control subjects were included in the dataset if the patient was not entered into the EMR system under more than one unique identifier and had at least one follow-up visit. Additionally, subjects were not included in the data set if during chart review the patient's ulcer was found to have resulted from traumatic or burn injury, HIV positive, history of critical limb ischemia, sickle cell disease, liver failure, cancer, or the patient was aged less than 18 years.

\section{Follow-up and Clinical Outcome}

Ulcer closure was the outcome of interest for this study. Ulcer closure was a dichotomous variable with healed and unhealed as the variables. The study period was from the first ulcer clinic visit date, or initial date of BBSS application, to day 180 after the first day (censored). A case report form (CRF) was designed and generated to help the reviewer capture data from the electronic medical record (EMR).

\section{Database description}

Data from this study were contributed to a university based medical center data warehouse where data from the hospital and clinic settings are integrated into a single relational data warehouse. These data include registration, outpatient, inpatient, operating room, emergency department, infection, appointment and anaesthesia data. Researchers with IRB and Health Insurance Portability and Accountability Act (HIPAA) approval requested data access to a limited dataset, which was approved for use.

A request for data was submitted to the data warehouse after approval was obtained. The data request was made for record dates from June 1st 2006 through January 1st of 2011. Patient level demographic data requested included date of birth (DOB), gender, race, and zip code.

Problem level data requested included relevant medical conditions including: ulcer type, infection type, diabetes, arterial disease, anemia, stroke, hypertension, congestive heart disease, rheumatoid arthritis, osteoarthritis, cancer, autoimmune disease, deep vein thrombosis, edema, lipodermatosclerosis, varicosities, and dermatitis. Visit level data requested included: visit type, date of visit, diagnosis, admission date, discharge date, procedure type, procedure date, laboratory test date and laboratory test result. Data from the data warehouse was received in Microsoft Excel spread sheet format.

Chart review data were collected first on paper case report forms and then input into a Microsoft Access Database. Problem level and visit level data were collected on variables that did not appear in the data warehouse. These variables were: wound size, wound classification, wound characteristics, amputation level, deformity, and secondary ulcer.

Additionally, a review of procedures was conducted to ensure inclusion of secondary procedures as well as a review of ulcer type to ensure proper classification of ulcer. Data analysis was performed on de-identified data translated from Microsoft Excel spread sheet and Microsoft Access database format into SAS programmable format (Table 1).

\begin{tabular}{|c|c|c|c|c|}
\hline & & Control $(n=158)$ & Apligraf users ( $n=126)$ & P-value \\
\hline Age, mean (SD) & & $59.4(12.9)$ & $62.1(16.2)$ & $>0.05$ \\
\hline \multicolumn{5}{|l|}{ Gender, N (\%) } \\
\hline & Male & $80(64.00)$ & $74(60.16)$ & \\
\hline & Female & $48(36.01)$ & $49(39.84)$ & $>0.05$ \\
\hline \multicolumn{5}{|l|}{ Race, N (\%) } \\
\hline & White & 61 (48.39) & $59(46.8)$ & \\
\hline & Black & $46(35.48)$ & $38(30.2)$ & \\
\hline & Hispanic & $10(8.06)$ & $22(17.46)$ & $>0.05$ \\
\hline Ulcer area, $\mathrm{cm}^{2}$, mean (std) & & $7.313(9.76)$ & $13.7(25.26)$ & $<0.05$ \\
\hline \multicolumn{5}{|l|}{ Ulcer grade $\mathrm{N}(\%)$} \\
\hline & I-II & $75(85.23)$ & $89(90.82)$ & \\
\hline
\end{tabular}


Citation: Yao M, Allen L, Diehl K, French M, Park N, Driver VR (2016) Retrospective Cohort Study Evaluating Clinical Outcomes in Lower Extremity Ulcers Treated with a Bi-layered Bioengineered Skin Substitute (BBSS) as Compared to Standard Therapy. Gen Med (LoS Angeles) 4: 282. doi:10.4172/2327-5146.1000282

Page 3 of 6

\begin{tabular}{|c|c|c|c|c|}
\hline & III-IV & $13(14.77)$ & $9(9.18)$ & $>0.05$ \\
\hline \multicolumn{5}{|l|}{ Type of ulcer, $\mathrm{N}(\%)$} \\
\hline & Diabetic ulcers & $91(57.59)$ & $92(73.02)$ & $<0.05$ \\
\hline & Arterial ulcers & $15(9.49)$ & $32(25.4)$ & $<0.05$ \\
\hline & Venous ulcers & $26(16.46)$ & $34(26.98)$ & $<0.05$ \\
\hline \multicolumn{5}{|l|}{ Type of co-morbidities } \\
\hline Hypertension (\%) & & $83(51.23)$ & $86(68.25)$ & $<0.05$ \\
\hline Coronory heart disease, N (\%) & & $25(19.20)$ & $27(21.4)$ & $>0.05$ \\
\hline Cerebrovascular disease, $\mathrm{N}(\%)$ & & 0 & $13(10.32)$ & $<0.05$ \\
\hline Diabetes, N (\%) & & $98(62.03)$ & $97(76.98)$ & $<0.05$ \\
\hline Peripherial arterial disease, $\mathrm{N}(\%)$ & & $35(22.15)$ & $47(37.3)$ & $<0.05$ \\
\hline Chronic renal disease, $\mathrm{N}(\%)$ & & 0 & $34(26.98)$ & $<0.05$ \\
\hline Congestive heart disease, $\mathrm{N}(\%)$ & & $12(7.59)$ & $21(16.67)$ & $<0.05$ \\
\hline History of minor amputation, $\mathrm{N}$ (\%) & & $22(13.93)$ & $30(23.81)$ & $<0.05$ \\
\hline History of major amputation, N (\%) & & $7(4.32)$ & $19(15.08)$ & $<0.05$ \\
\hline \multirow[t]{7}{*}{ Number of co-morbidies } & 0 & $48(29.63)$ & $9(7.14)$ & \\
\hline & 1 & $25(15.82)$ & $22(17.46)$ & \\
\hline & 2 & $50(31.65)$ & $32(25.40)$ & \\
\hline & 3 & $28(17.72)$ & $21(16.67)$ & \\
\hline & 4 & $7(4.43)$ & $22(17.46)$ & \\
\hline & 5 & $4(2.53)$ & $10(7.94)$ & \\
\hline & 6 & $0(0.00)$ & $10(7.94)$ & $<0.05$ \\
\hline Average co-morbidities, mean (std) & & $1.62(1.312)$ & $2.75(1.66)$ & $<0.05$ \\
\hline
\end{tabular}

Table 1: Baseline characteristics of patients.

\section{Statistical Analysis}

We performed descriptive statistics on the BBSS user cohort. Continuous variables of both BBSS and control groups were compared with a student $t$ test. Categorical variables were analyzed using Chisquare. Incidence rate (IR) of wound healing with confidence intervals (CI) were calculated for all patients with ulcers and then stratified by ulcer type. To adjust for all potential confounders noted via chart review (diabetes, peripheral arterial disease, chronic kidney disease, coronary heart disease, etc.).

We constructed multivariate-adjusted COX proportional hazard models to evaluate dichotomous ulcer healing outcomes. Additionally, we conducted subgroup analyses in BBSS subjects by examining whether timing of application after the onset of an index ulcer was associated with outcomes using a separate confounder-adjusted COX proportional hazard model. Specifically, we compared overall healing rate (Table 2), time to achieve wound closure, healed wounds per 100 person-years with and without BBSS application, incidence rates of wound healing by ulcer type, unadjusted and adjusted hazard ratios of wound healing.

\begin{tabular}{|c|c|c|c|}
\hline & $\begin{array}{l}\text { Unhealed/censored, } \\
\mathbf{N}(\%)\end{array}$ & $\begin{array}{l}\text { Healed, } \\
\mathbf{N}(\%)\end{array}$ & $\begin{array}{l}\text { Difference } \\
(\mathbf{A}-\mathbf{C}) \%\end{array}$ \\
\hline All types of ulcers & & $67(42.41)$ & \\
\hline Control (N=158) & $91.57(57.59)$ & $88(69.86)^{*}$ & 27.4 \\
\hline Aapligraf (N=126) & $40(31.75)$ & $38(41.76)$ & \\
\hline Diabetic ulcer & $53(58.24)$ & $61(66.30)^{*}$ & 24.6 \\
\hline Control (N=91) & $31(33.70)$ & & \\
\hline Apligraf (N=92) & $10(66.67)$ & $5(33.33)$ & \\
\hline Arterial ulcer & $15(46.88)$ & $17(53.13)$ & \\
\hline Control (N=15) & & & \\
\hline Apligraf (N=32) & & & \\
\hline Venous ulcer & & & \\
\hline
\end{tabular}


Citation: Yao M, Allen L, Diehl K, French M, Park N, Driver VR (2016) Retrospective Cohort Study Evaluating Clinical Outcomes in Lower Extremity Ulcers Treated with a Bi-layered Bioengineered Skin Substitute (BBSS) as Compared to Standard Therapy. Gen Med (Los Angeles) 4: 282. doi:10.4172/2327-5146.1000282

Page 4 of 6

\begin{tabular}{|c|c|c|c|}
\hline Control $(\mathrm{N}=26)$ & $12(46.15)$ & $15(57.69)$ & \\
\hline Apligraf $(\mathrm{N}=34)$ & $10(29.41)$ & $25(73.58)$ & 15.9 \\
\hline${ }^{*} \mathrm{P}<0.05$ compared to control \\
\hline
\end{tabular}

Table 2: Healing Rate between apligraf and control.

\section{Results}

There were 126 patients treated with BBSS and 162 in the control group. Average age was $62.1 \pm 16.2$ and $59.4 \pm 12.9$, respectively. Regarding baseline wound area between two groups, the BBSS group was significantly larger than the non BBSS group (13.7 vs. $7.3 \mathrm{~cm}^{2)}$, $\mathrm{P}<0.05$. Not only prevalence of those subjects with co-morbidities, but also average numbers of co-morbidities were significantly greater in the BBSS group than the control group ( 2.75 vs. $1.62, \mathrm{P}<0.05)$. Overall, more ulcers healed in the BBSS group than in the control group (69.84\% vs. $41.98 \%, \mathrm{P}<0.05)$, indicating BBSS yielded a $27.9 \%$ healing

benefit as compared to standard of care. Meanwhile, among all healed ulcers within 180 days, average time to achieve wound closure was 70.1 days in the BBSS group and 118.03 days in the control, indicating that the BBSS healed ulcers 48 days earlier than that if standard of care.

In all types of ulcers, the IR of wound closure was 251.5 per 100 person year (95\% CI is 204.2-309.9) in the BBSS group and 157.76 per 100 person year in the control group (95\% CI is 124.2-200.4) (Table 3). After adjusting for potential confounders (diabetes, peripheral arterial disease, chronic kidney disease, coronary heart disease, etc).

We constructed multivariate-adjusted COX proportional hazard models to evaluate dichotomous ulcer healing outcomes. BBSS was 1.8 times more likely to heal wounds (regardless of ulcer type) than that of standard of care (HR=1.76, 95\% CI 1.14-2.73) and 1.56 times more likely to heal the diabetic ulcers than that of standard of care $(\mathrm{HR}=1.56,95 \% \mathrm{CI} 0.89-2.71)$. This study showed that, despite the greater significant co-morbidities, subjects treated with BBSS healed faster (Table 3).

\begin{tabular}{|c|c|c|c|c|c|c|c|}
\hline Ulcer type & Group & Total $\mathbf{N}$ & N Event & $\begin{array}{l}\text { Total follow-up time } \\
\text { (PY) }\end{array}$ & IR/100PY & IR 95\% Cl_lower & IR 95\% Cl_high \\
\hline \multicolumn{8}{|l|}{ All ulcers } \\
\hline & Control & 158 & 67 & 42.473 & 157.76 & 124.17 & 200.44 \\
\hline & Apligraf & 126 & 88 & 34.99 & 251.5 & 204.18 & 309.94 \\
\hline \multicolumn{8}{|c|}{ Diabetic ulcer } \\
\hline & Control & 91 & 32 & 20.97 & 181.21 & 131.85 & 249.04 \\
\hline & Apligraf & 92 & 61 & 26.01 & 234.53 & 182.48 & 301.43 \\
\hline \multicolumn{8}{|c|}{ Arterial ulcer } \\
\hline & Control & 15 & 5 & 3.18 & 157.23 & 65.41 & 377.76 \\
\hline & Apligraf & 32 & 17 & 11.27 & 150.84 & 93.77 & 242.64 \\
\hline \multicolumn{8}{|c|}{ Venous ulcer } \\
\hline & Control & 26 & 15 & 6.5 & 230.77 & 139.12 & 382.79 \\
\hline & Apligraf & 34 & 25 & 9.35 & 267.38 & 180.67 & 395.71 \\
\hline \multicolumn{8}{|c|}{${ }^{*} \mathrm{PY}=$ Person year } \\
\hline
\end{tabular}

Table 3: Incidence rate of wound healing.

\section{Discussion}

In this study, BBSS application reduced ulcer time to heal (Table 4) by 46 days and net healing benefit was $27.4 \%$. We compared our results with two RCTs $[4,11]$. An RCT with BBSS treatment for diabetic foot ulcers (DFU) on 208 patients with non-ischemic, chronic plantar diabetic foot ulcers done by Veves et al., showed that, at 12 weeks, 56\% of the BBSS treated patients achieved complete wound healing in comparison with $38 \%$ of the control group, with the median time to complete closure being 65 days for the BBSS treated group and 90 days in the control group.
Another multi-center study, conducted by Falanga et al., on 293 patients in an outpatient setting examined venous leg ulcers (VLU) receiving either compression therapy alone compared with compression therapy and serial (up to 5) applications of the human skin equivalent, BBSS endpoint of 6 months, with $63 \%$ vs. $49 \%$ of patients having completely healed, respectively, and the median time to complete wound closure was 61 vs. 181 days, respectively. The treatment results for this study were comparable with results obtained from the above mentioned RCTs. 
Citation: Yao M, Allen L, Diehl K, French M, Park N, Driver VR (2016) Retrospective Cohort Study Evaluating Clinical Outcomes in Lower Extremity Ulcers Treated with a Bi-layered Bioengineered Skin Substitute (BBSS) as Compared to Standard Therapy. Gen Med (LoS Angeles) 4: 282. doi:10.4172/2327-5146.1000282

Page 5 of 6

\begin{tabular}{|c|c|c|c|}
\hline & Healed ulcer (N) & Days to achieve wound closure, mean \pm std) & Difference (A-C) days \\
\hline \multicolumn{4}{|l|}{ All types of ulcers } \\
\hline Control $(\mathrm{N}=158)$ & 57 & $116.90 \pm 59.45$ & \\
\hline Aapligraf $(\mathrm{N}=126)$ & 84 & $70.70 \pm 50.92^{*}$ & 46.2 \\
\hline \multicolumn{4}{|l|}{ Diabetic ulcer } \\
\hline Control $(\mathrm{N}=89)$ & 32 & $115.8 \pm 57.61$ & \\
\hline Apligraf ( $\mathrm{N}=91)$ & 60 & $65.28 \pm 51.33^{*}$ & 50.2 \\
\hline \multicolumn{4}{|l|}{ Arterial ulcer } \\
\hline Control $(N=15)$ & 5 & $134.0 \pm 48.65$ & \\
\hline Apligraf ( $\mathrm{N}=32)$ & 17 & $83.05 \pm 59.23$ & 50.9 \\
\hline \multicolumn{4}{|l|}{ Venous ulcer } \\
\hline Control $(\mathrm{N}=19)$ & 12 & $99.0 \pm 63.08$ & \\
\hline Apligraf $(\mathrm{N}=34)$ & 23 & $78.13 \pm 52.31$ & 20.9 \\
\hline
\end{tabular}

Table 4: Time to achieve would closure between Apligraf and control in all healed patients.

Treatment of chronic LEUs requires an interdisciplinary approach and often requires advanced wound therapy which can contribute to considerable health care cost [12]. Driver et al., [13] reported that costs for treating the highest grade ulcers are 8 times higher than for those treating low grade ulcers. Patients with DFUs require more frequent emergency department visits and are more commonly admitted to the hospital, requiring longer lengths of stay [14].

Limb salvage efforts may include aggressive therapy such as revascularization procedures and advanced wound healing modalities [15]. Zaulyanov et al., reviewed several clinical studies on both DFU and VLU with BBSS treatment. BBSS has been determined as more effective and economical at healing chronic VLU [16-18] and DFU [19] than standard wound care therapy alone [19]. BBSS has been shown to reduce the risk of development of osteomyelitis and amputation in diabetic patients (Table 5).

\begin{tabular}{|l|c|c|}
\hline & Unadjusted HRs (95\% Cl) & Adjusted HRs (95\% CI) \\
\hline All ulcers & $1.77(1.26-2.48)$ & $1.69(1.14-2.73)$ \\
\hline Type of ulcers & & \\
\hline Diabetic ulcers & $1.46(0.94-2.23)$ & $1.51(0.91-2.49)$ \\
\hline Arterial ulcers & $0.86(0.29-2.26)$ & $0.37(0.09-1.51)$ \\
\hline Venous ulcers & $1.33(0.66-2.69)$ & $1.11(0.50-2.45)$ \\
\hline${ }^{*}$ control is reference group
\end{tabular}

Table 5: Unadjusted and Adjusted Hazard Ratio (HRs) of Ulcers in patients with and without Apligraf.

\section{Conclusions}

As with most observational studies, we realize the limitations of our study. This is a retrospective cohort study using administrative claim data and the electronic medical record as our data source. Administrative claim data and electronic medical records are mainly for clinical practice use which may include incomplete documentation, unrecoverable or unrecorded information, difficultly in interpreting and verifying some information, and also variances in the quality of information recorded by individual medical professionals [20-22]. This study matched controls to case subjects based on age and gender. However, no matching occurred for any additional baseline differences between controls and case subjects. All of these limitations are due to the 'nature' of the data source, which can generate additional bias and confounders in our final findings.

In conclusion, BBSS may be effective in accelerating wound healing in patients with severe complex lower extremity ulcers and comorbidities in the pragmatic continuum of foot care.

\section{References}

1. Singh N, Armstrong DG, Lipsky BA (2005) Preventing foot ulcers in patients with diabetes. JAMA 293: 217-228.

2. Zaulyanov L, Kirsner RS (2007) A review of a bi-layered living cell treatment (Apligraf) in the treatment of venous leg ulcers and diabetic foot ulcers. Clinical Interventions in Aging 2: 93-98.

3. Falanga V, Margolis D, Alvarez O (1998) Rapid healing of venous ulcers and lack of clinical rejection with an allogeneic cultured human skin equivalent. Human Skin Equivalent Investigators Group. Archives of Dermatology134: 293-300.

4. Veves A, Falanga V, Armstrong DG, Sabolinski ML (2001) Graftskin, a human skin equivalent, is effective in the management of noninfected neuropathic diabetic foot ulcers: a prospective randomized multicenter clinical trial. Diabetes care. Feb 24: 290-295. 
Citation: Yao M, Allen L, Diehl K, French M, Park N, Driver VR (2016) Retrospective Cohort Study Evaluating Clinical Outcomes in Lower Extremity Ulcers Treated with a Bi-layered Bioengineered Skin Substitute (BBSS) as Compared to Standard Therapy. Gen Med (LoS Angeles) 4: 282. doi:10.4172/2327-5146.1000282

Page 6 of 6

5. Edmonds M (2009) Apligraf in the treatment of neuropathic diabetic foot ulcers. The international journal of lower extremity wounds.8: 11-18.

6. Concato J, Shah N, Horwitz RI (2000) Randomized, controlled trials, observational studies, and the hierarchy of research designs. $\mathrm{N}$ Engl J Med. Jun 22 342: 1887-1892.

7. Horn SD, Gassaway J (2007) Practice-based evidence study design for comparative effectiveness research. Med Care 45: S50-57.

8. Gottrup F, Apelqvist J, Price P (2010) Outcomes in controlled and comparative studies on non-healing wounds: recommendations to improve the quality of evidence in wound management. J Wound Care 19: 237-268.

9. Carter MJ, Fylling CP, Li WW (2011) Analysis of run-in and treatment data in a wound outcomes registry: clinical impact of topical platelet-rich plasma gel on healing trajectory. International Wound Journal 8: 638-650.

10. Bagshaw SM, Bellomo R (2008) The need to reform our assessment of evidence from clinical trials: a commentary. Philos Ethics Humanit Med $3: 23$.

11. Brem H, Balledux J, Bloom T, Kerstein MD, Hollier L (2000) Healing of diabetic foot ulcers and pressure ulcers with human skin equivalent: a new paradigm in wound healing. Arch Surg 135: 627-634.

12. Driver VR, Madsen J, Goodman RA (2005) Reducing amputation rates in patients with diabetes at a military medical center: the limb preservation service model. Diabetes Care 28: 248-253.

13. Driver VR, Fabbi M, Lavery LA, Gibbons G (2010) The costs of diabetic foot: the economic case for the limb salvage team. J Vasc Surg 52: $17 \mathrm{~S}-22 \mathrm{~S}$.
14. Stockl K, Vanderplas A, Tafesse E, Chang E (2004) Costs of lowerextremity ulcers among patients with diabetes. Diabetes Care 27:2129-2134.

15. Driver VR, Yao M (2011) The economics of limb salvage in diabetes. Plast Reconstr Surg 127 :296S-297S.

16. Curran MP, Plosker GL (2003) Bilayered bioengineered skin substitute (Apligraf): a review of its use in the treatment of venous leg ulcers and diabetic foot ulcers. BioDrugs: clinical immunotherapeutics, biopharmaceuticals and gene therapy 16: 439-455.

17. Fivenson D, Scherschun L (2003) Clinical and economic impact of Apligraf for the treatment of nonhealing venous leg ulcers. International Journal of Dermatology 42 : 960-965.

18. Schonfeld WH, Villa KF, Fastenau JM, Mazonson PD, Falanga V (2000) An economic assessment of Apligraf (Graftskin) for the treatment of hard-to-heal venous leg ulcers. Wound Repair Regen 8: 251-257.

19. Redekop WK, McDonnell J, Verboom P, Lovas K, Kalo Z (2003) The cost effectiveness of Apligraf treatment of diabetic foot ulcers. Pharmaco Economics 21: 1171-1183.

20. Worster A, Haines T (2004) Advanced statistics: understanding medical record review (MRR) studies. Acad Emerg Med11: 187-192.

21. Wu L, Ashton CM (1997) Chart review. A need for reappraisal. Eval Health Prof. Jun 20: 146-163.

22. vonKoss Krowchuk H, Moore ML, Richardson L (1995) Using health care records as sources of data for research. J Nurs Meas $3: 3-12$. 